\title{
Ganetespib induces G2/M cell cycle arrest and apoptosis in gastric cancer cells through targeting of receptor tyrosine kinase signaling
}

\author{
HARRY LEE, NIPUN SAINI, AMANDA B. PARRIS, MING ZHAO and XIAOHE YANG \\ Julius L. Chambers Biomedical/Biotechnology Research Institute, Department of Biological and \\ Biomedical Sciences, North Carolina Central University, Kannapolis, NC 28081, USA
}

Received March 22, 2017; Accepted July 6, 2017

DOI: $10.3892 /$ ijo.2017.4073

\begin{abstract}
Heat shock protein 90 (HSP90) regulates several important cellular processes via its repertoire of "client proteins'. These client proteins have been found to play fundamental roles in signal transduction, cell proliferation, cell cycle progression and survival, as well as other features of malignant cells, such as invasion, tumor angiogenesis and metastasis. Thus, HSP90 is an emerging target for cancer therapy. To this end, we evaluated ganetespib (STA9090), a novel and potent HSP90 inhibitor, for its activity in gastric cancer cell lines. Ganetespib significantly inhibited the proliferation of AGS and N87 human gastric cancer cell lines and potently induced $\mathrm{G} 2 / \mathrm{M}$ cell cycle arrest and apoptosis. Upregulation of cleaved poly(ADP-ribose) polymerase (c-PARP), c-caspase-3, c-caspase- 8 and c-caspase- 9 and suppression of gastric cancer-associated HSP90 client proteins, including ErbB2, Erk, Akt, mTOR, GSK3 and Src, were observed in ganetespib-treated cells. These findings demonstrate that the ganetespib-induced mechanism of cell growth inhibition involves the activation of death receptor and mitochondrial pathways and the inhibition of receptor tyrosine kinase signaling pathways. Our study implicates ganetespib as a potential strategy for gastric cancer treatment, which warrants further preclinical and clinical investigation.
\end{abstract}

\section{Introduction}

Gastric cancer(GC) is the second leading cause of cancer-related deaths worldwide and imposes a major health burden due to its

Correspondence to: Dr Xiaohe Yang, Julius L. Chambers Biomedical/Biotechnology Research Institute, Department of Biological and Biomedical Sciences, North Carolina Central University, 500 Laureate Way, NRI 4301, Kannapolis, NC 28081, USA

E-mail: xyang@nccu.edu

Key words: ganetespib, HSP90, gastric cancer, G2/M arrest, apoptosis, receptor tyrosine kinase signaling poor prognosis (1). Although surgery is a relatively effective treatment option for GC, the 5-year survival rate is only $29 \%$ and the median survival rate is less than a year (2). Various cytotoxic drugs, such as 5-fluorouracil, platinum and taxane, are treatment options, but drug resistance is significant. Thus, the effective management of advanced GC remains a significant challenge, especially for aggressive subtypes (3), making the development of novel therapies targeting refractory and resistant cases an urgent need.

The expression of various oncogenic growth factors, such as epidermal growth factor receptor (EGFR) and ErbB2, is elevated in gastrointestinal carcinomas and is associated with poor prognosis and reduced overall survival (4-6). The overexpression of these proteins further activates downstream signaling pathways, such as phosphatidylinositol 3-kinase (PI3K)/Akt and mitogen-activated protein kinase (MAPK)/Erk pathways, that mediate oncogenic cellular proliferation, differentiation, angiogenesis, tumor metastasis and survival (7). These proteins are client proteins of heat shock protein 90 (HSP90) which help facilitate them to escape normal proteolytic turnover and contribute to tumor development and survival (4). Moreover, the overexpression of HSP90 and its client proteins has been associated with the development of GC and its clinicopathological features, such as tumor size, lymph node metastases and patient survival (8). Hence, targeting HSP90 and, indirectly, its associated oncogenic client proteins is a promising anti-GC therapeutic strategy.

Ganetespib (STA-9090) is a resorcinol-based second generation HSP90 inhibitor with enhanced potency and a favorable safety profile as compared to geldanamycin derivatives (9). In preclinical studies, ganetespib has demonstrated significant inhibition of cell proliferation and tumor growth in cell and xenograft models of multiple cancers (9). Currently a number of ongoing clinical trials are investigating the effect of ganetespib in various cancers, including rectal, ErbB2 ${ }^{+}$metastatic breast, multiple myeloma and lung cancers (10). Recently, Liu et al reported the efficacy of ganetespib in targeting EGFR-mediated GC in cell lines and xenograft models (11). Still, further preclinical studies are necessary to fully elucidate other receptor tyrosine kinase (RTK) signaling pathways that may be involved in ganetespib-mediated inhibition of GC. In 
the present study, we demonstrate the efficacy of ganetespib in targeting multiple oncogenic pathways associated with RTK signaling in GC cells. Given the poor clinical outcomes associated with growth factor-mediated GC and the lack of effective GC therapeutics, ganetespib has the potential to be developed into a therapeutic agent for GC.

\section{Materials and methods}

Materials. Ganetespib was purchased from Medkoo Biosciences, Inc. (Chapel Hill, NC, USA). Primary antibodies specific to Cyclin B1, cleaved caspase-3, cleaved caspase-8, cleaved caspase-9, cleaved PARP, Akt, phospho Akt (pAkt), mTOR, pmTOR, ErbB2, pErbB2, GSK3, pGSK3, Erk, pErk, Src and pSrc were purchased from Cell Signaling Technology (Danvers, MA, USA); and cyclin D1, cyclin E, Cdk1, E2F1, p27, survivin, caspase-8, caspase-9, EGFR and $\beta$-actin were purchased from Santa Cruz Biotechnology (Santa Cruz, CA, USA). Secondary anti-mouse or anti-rabbit antibodies were purchased from Thermo Scientific (Rockford, IL, USA).

Cell culture. Human AGS and N87 GC cell lines were purchased from the American Type Culture Collection (ATCC; Manassas, VA, USA). Cells were maintained in Dulbecco's modified Eagle's medium (DMEM) (Life Technologies; Carlsbad, CA, USA) supplemented with $10 \%$ fetal bovine serum, $100 \mu \mathrm{g} / \mathrm{ml}$ penicillin and $100 \mu \mathrm{g} / \mathrm{ml}$ streptomycin at $37^{\circ} \mathrm{C}$ in a humidified incubator with a $5 \% \mathrm{CO}_{2}$ atmosphere.

MTT assay. MTT (3-(4, 5-dimethylthiazol-2-yl)-2, 5-diphenyltetrazolium bromide) assay (Sigma-Aldrich, St. Louis, MO, USA) was used to determine the cytotoxicity of ganetespib in different cell lines. AGS and N87 cells were seeded at a density of 1000 cells/well in a 96-well plate and incubated overnight. After 5 days of ganetespib treatment $(0,0.5,1,5$, $10,50$ or $100 \mathrm{nM})$, MTT was added to the cells at a final concentration of $0.5 \mathrm{mg} / \mathrm{ml}$. After $3 \mathrm{~h}$ of incubation with MTT, media was aspirated and $50 \mu 1$ of DMSO was added to each well and kept on a shaker for $45 \mathrm{~min}$. The absorbance at $570 \mathrm{~nm}$ was then measured using a SynergyMx microplate reader (BioTek; Winooski, VT, USA). All samples were analyzed in five replicates. $\mathrm{IC}_{50}$ was calculated manually by linear interpolation using the formula: $\mathrm{IC}_{50}=[(50-\mathrm{A}) /(\mathrm{B}-\mathrm{A})]$ $\mathrm{x}(\mathrm{D}-\mathrm{C})+\mathrm{C}$, where $\mathrm{A}=$ the first point on the curve, expressed as percent inhibition, that is less than $50 \%$; $\mathrm{B}=$ the first point on the curve, expressed as percent inhibition, that is greater than or equal to $50 \%$; $\mathrm{C}=$ the concentration of inhibitor that gives $\mathrm{A} \%$ inhibition; and $\mathrm{D}=$ the concentration of inhibitor that gives $\mathrm{B} \%$ inhibition (12).

Clonogenic assay. Viable AGS and N87 cells were plated at a density of 300 cells/well and 1000 cells/well, respectively, and allowed to adhere overnight. Cells were treated with ganetespib $(0,1.25,2.5,5$ or $10 \mathrm{nM})$ for 10 days. After 10 days, colonies were washed twice with phosphate buffered saline (PBS) and stained with $0.5 \%$ crystal violet $\left(1: 1\right.$ methanol: $\left.\mathrm{H}_{2} \mathrm{O}\right)$ for $30 \mathrm{~min}$ at room temperature. The extra stain was aspirated, and the plates were washed with tap water and air dried. Colonies were counted and imaged with a digital camera mounted on a Nikon C-LEDS microscope.
Cell cycle analysis. AGS and N87 cells were treated with ganetespib (0 and $500 \mathrm{nM}$ ) for $24 \mathrm{~h}$ in complete medium. Floating and adherent cells were collected, washed twice with cold PBS and centrifuged. Cells were then fixed in $70 \%(\mathrm{v} / \mathrm{v})$ ethanol at $-20^{\circ} \mathrm{C}$. After centrifugation, cells were washed with cold PBS and stained with propidium iodide (PI) in RNase solution. After incubation for $45 \mathrm{~min}$ at $37^{\circ} \mathrm{C}$, cells were strained with a $40 \mu \mathrm{m}$ filter and the cell cycle distribution was determined using a Guava EasyCyte 8 Flow Cytometer (Millipore; Billerica, MA, USA).

Apoptosis assay. Apoptosis was measured using an Annexin-V-Fluos Staining kit (Sigma-Aldrich) according to the manufacturer's protocol. Briefly, the AGS and N87 cells were treated with ganetespib $(0,25$ or $50 \mathrm{nM})$ for $24 \mathrm{~h}$. Then, the cells were harvested and washed with cold PBS. After staining with Annexin V-FITC/PI, the percentage of apoptotic cells was quantified using a Guava EasyCyte 8 Flow Cytometer (Millipore).

Western blotting. The protein concentrations of ganetespibtreated AGS and N87 cell extracts were determined using the BCA assay (Thermo Scientific). Protein from each sample $(50 \mu \mathrm{g})$ was resolved using SDS-PAGE and then transferred to a nitrocellulose membrane. The membranes were blocked in 5\% non-fat milk in Tris-buffered saline with Tween (TBST) for $1 \mathrm{~h}$, followed by overnight incubation in appropriate primary antibodies at $4^{\circ} \mathrm{C}$. After washing, membranes were incubated in species-specific horseradish peroxidase-conjugated secondary antibody for $1 \mathrm{~h}$ at room temperature. After further washing, specific protein bands were detected with enhanced chemiluminescence reagents and imaged with a FluorChemE imager (Cell Biosciences; Santa Clara, CA, USA). $\beta$-actin was used as a loading control.

Quantitative RT-PCR ( $q R T-P C R$ ). Total RNA was extracted from the treated cells and purified using an RNeasy mini kit (Qiagen; Germantown, MD, USA), as per the manufacturer's instructions. Purified RNA $(1 \mu \mathrm{g})$ was used to synthesize cDNA using iScript cDNA Synthesis kit (Bio-Rad; Hercules, CA, USA). The qPCR assay of triplicate samples was carried out on a Bio-Rad CFX96 system using SYBR green (Bio-Rad). The primer sequences used are as follows: CCNB1-forward primer (F)-GACAACTTGAGGAAGAGCAAGC, reverse primer (R)-ATGGTCTCCTGCAACAACCT; CCND1F-GGCGGATTGGAAATGAACTT, R-TCCTCTCCAAA ATGCCAGAG; CCNE1-F-GAAATGGCCAAAATCGA CAG, R-TCTTTGTCAGGTGTGGGGA; CDK4-F-GTCG GCTTCAGAGTTTCCAC, R-TGCAGTCCACATATGCA ACA; CDKN1B-F-TGGAGAAGCACTGCAGAGAC, R-GCG TGTCCTCAGAGTTAGCC; and $\beta$-actin-F-GCACCACA CCTTCTACAATGAGC, R-GACGTAGCACAGCTTCTCC TTAATG. Relative mRNA levels were quantified based on the cycle threshold $(\mathrm{Ct})$ values of the tested genes as normalized to the control $\beta$-actin gene.

Statistical analysis. A Student's t-test for comparison of two groups was used for statistical analysis. Calculations were performed using GraphPad Prism software (GraphPad; La Jolla, CA, USA) and data were expressed as means \pm standard error 
A

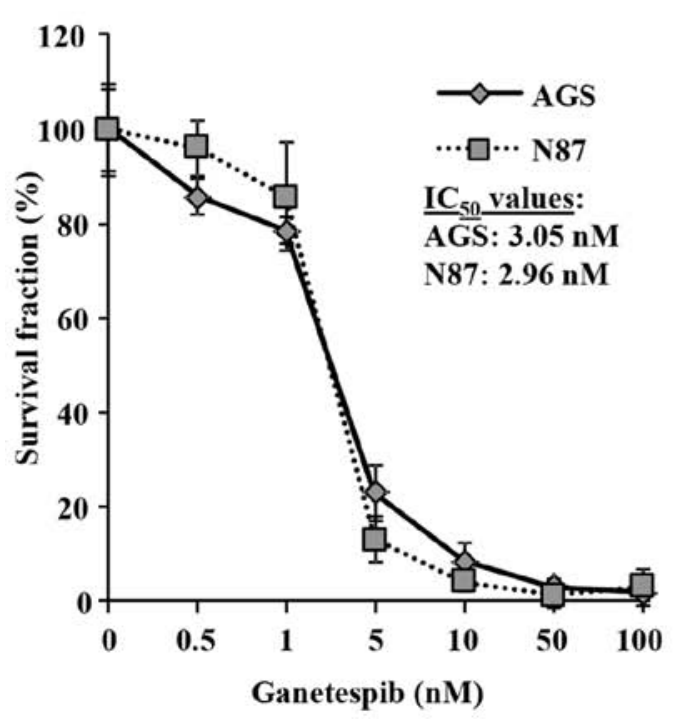

B

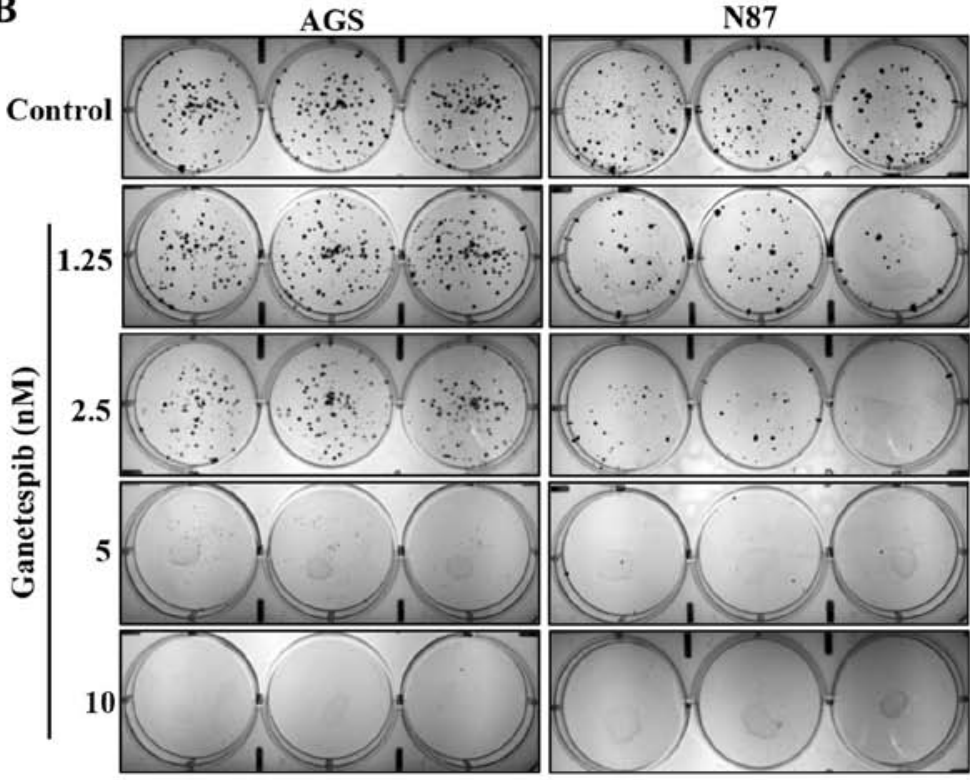

Figure 1. Ganetespib inhibits GC cell proliferation in vitro. (A) The cytotoxic potential of ganetespib was assessed in AGS and N87 cells after 5 days of ganetespib treatment $(0,0.5,1,5,10,50$ or $100 \mathrm{nM})$ using MTT assay. $\mathrm{IC}_{50}$ values were calculated as detailed in Materials and methods. (B) The growth potential of AGS and N87 cells in the presence of ganetespib $(0,1.25,2.5,5$ or $10 \mathrm{nM})$ for 10 days was assessed using clonogenic assay. Representative images of the crystal violet-stained colonies are shown.

of the mean (SEM) of at least three independent experiments. A P-value of $\leq 0.05$ was considered to indicate a statistically significant difference.

\section{Results}

Ganetespib inhibits proliferation and colony formation in AGS and N87 cell lines. Ganetespib inhibits the proliferation of various cancer cells at low nanomolar concentrations (9). To evaluate the effects of ganetespib on GC cell proliferation, we utilized AGS and N87 human GC cell lines that express high levels of EGFR (11) and ErbB2, respectively. AGS and N87 cells were treated with low concentrations of ganetespib $(0,0.5,1,5,10,50$ or $100 \mathrm{nM})$ for 5 days to determine its effect on cell proliferation. MTT assay revealed that ganetespib dose-dependently induced significant cell growth inhibition in both AGS and N87 cell lines with $\mathrm{IC}_{50}$ of 3.05 and $2.96 \mathrm{nM}$, respectively (Fig. 1A). To confirm our results, a clonogenic assay was performed on AGS and N87 cells treated with ganetespib $(0,1.25,2.5,5$, or $10 \mathrm{nM})$ for 10 days. Consistent with the MTT results, ganetespib also significantly inhibited colony formation at low concentrations ( 5 and $2.5 \mathrm{nM}$ ) in AGS and N87 cells, respectively (Fig. 1B). Together, these data indicate the anti-proliferative capacity of ganetespib in GC cell lines.

Ganetespib induces G2/M cell cycle arrest in AGS and N87 $G C$ cell lines. To further understand the anti-proliferative mechanism of ganetespib, we examined its effects on cell cycle progression in AGS and N87 cells. A high concentration of ganetespib was used in both cell lines to ensure significant inhibition of treated cells as compared to controls. Cell cycle analysis using flow cytometry indicated that ganetespib (500 $\mathrm{nM}$ for $24 \mathrm{~h}$ ) induced a significant reduction in $\mathrm{G} 0 / \mathrm{G} 1$ and $\mathrm{S}$ phase, which is indicative of a decreased proliferative cell population (Fig. 2A and B). Importantly, a significant accumulation of cells in G2/M phase was evident in both cell lines, but at different intensities. This suggests that ganetespib induces a specific pattern of cell cycle arrest in a cell linespecific manner and prevents entry into G0/G1 phase by potentially regulating the associated checkpoint regulators.

Various cell cycle regulators are involved in the progression of cells from one phase to another. To elucidate the mechanism of ganetespib-induced cell cycle arrest, we examined the expression levels of several key cell cycle regulators in cells treated with a range of ganetespib concentrations $(0-1000 \mathrm{nM})$ for $24 \mathrm{~h}$. In AGS and N87 cell lines, ganetespib downregulated the protein levels of E2F1, cyclin D1 and cyclin-dependent kinase 1 (Cdk1) (Fig. 2C). Notably, cyclin B1 and p27 were dose-dependently upregulated in both cell lines. This observation will be addressed in the Discussion. Consistent with the western blot data, qPCR analysis revealed that ganetespib also induces a significant increase in the mRNA levels of cyclin B1 (CCNB1) and p27 (CDKN1B) and a concomitant reduction in cyclin D1 (CCND1), cyclin E1 (CCNE1) and CDK4 mRNA expression in both cell lines (Fig. 2D). Taken together, these data suggest that ganetespib inhibits GC cell cycle progression by transcriptionally, as well as translationally, modifying key cell cycle regulators in vitro.

Ganetespib-treated AGS and N87 cells undergo apoptosis through the activation of mitochondrial and death receptor pathways. Apoptosis is a common mechanism of protein degradation associated with HSP90 inhibition (13). To better understand the mechanisms of ganetespib-induced cell death in AGS and N87 cells, we used flow cytometry to determine the degree of apoptosis. To this end, the cells were treated with 0-50 nM of ganetespib for $24 \mathrm{~h}$, followed by analysis of apoptotic cells using Annexin V/PI staining. Cells in the early apoptotic 
A
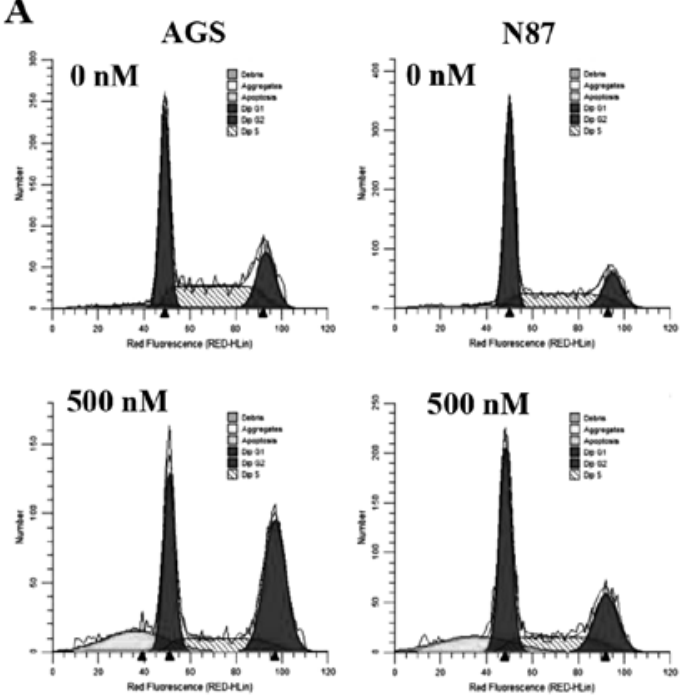

D

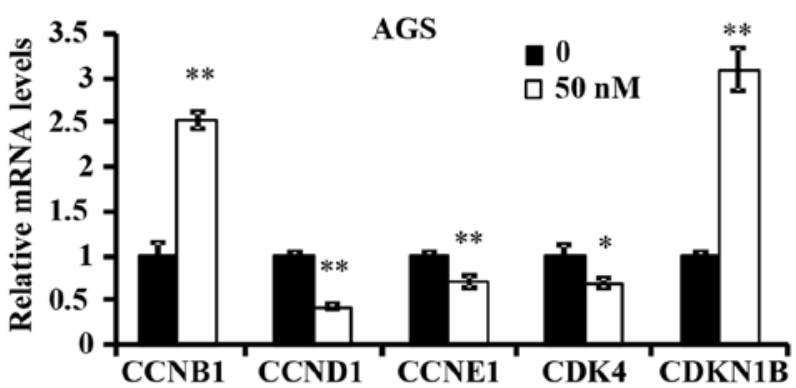

B
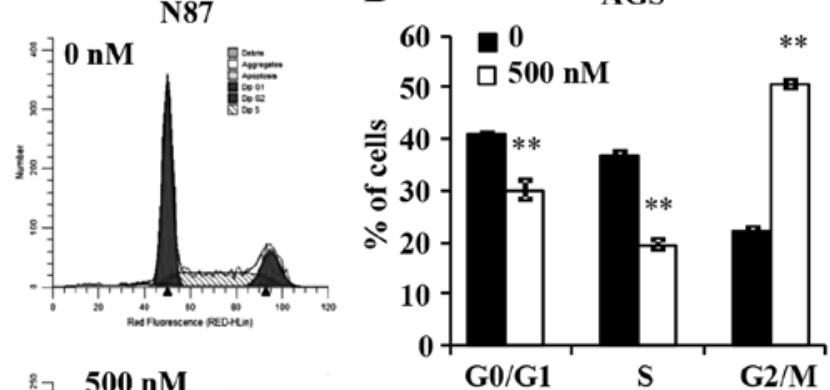

N87

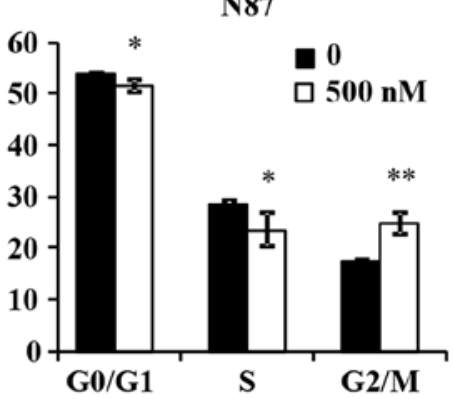

C

AGS

N87
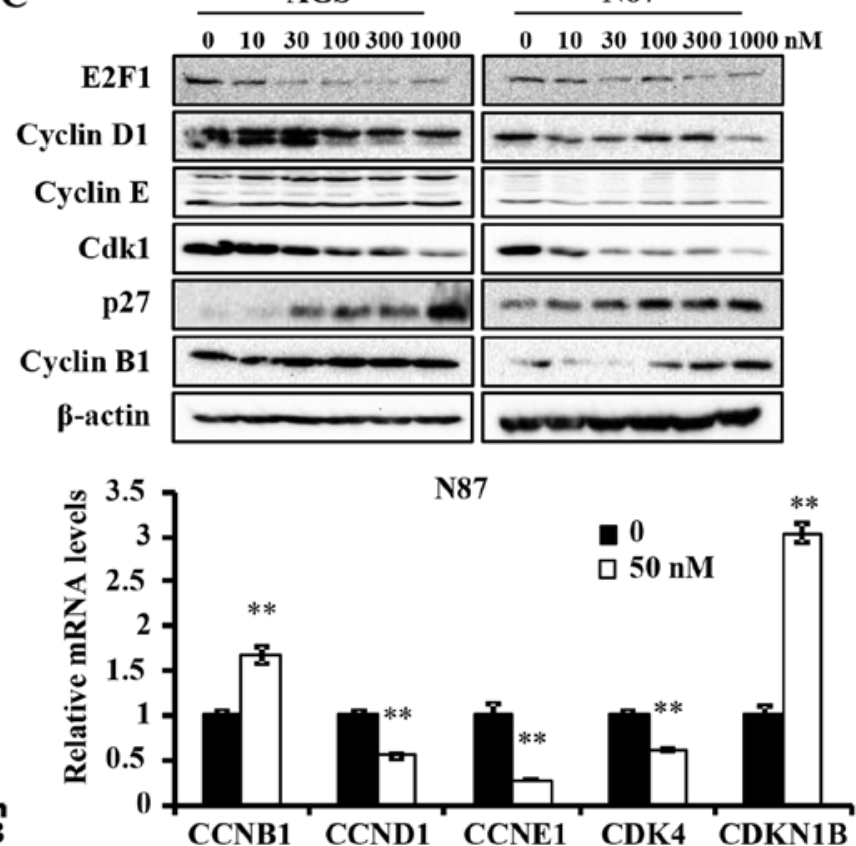

Figure 2. Ganetespib induces G2/M cell cycle arrest in GC cells. (A and B) AGS and N87 cells were treated with ganetespib ( 0 or $500 \mathrm{nM})$ for $24 \mathrm{~h}$, stained with PI and analyzed for cell cycle distribution using flow cytometry. Representative images of flow cytometry plots are shown in (A). The percentage of cells in G0/G1, S and G2/M are graphed in (B). (C) AGS and N87 cells were treated with ganetespib (0, 10, 30, 100, 300 or $1000 \mathrm{nM})$ for $24 \mathrm{~h}$, followed by western blot analysis of key cell cycle regulators. (D) Relative mRNA levels of the indicated cell cycle regulators were quantified in AGS and N87 cells treated with ganetespib $(0$ or $50 \mathrm{nM})$ for $24 \mathrm{~h}$. All values are graphed as the means \pm SEM $\left({ }^{*} \mathrm{P} \leq 0.05,{ }^{* *} \mathrm{P} \leq 0.01\right.$ as compared to untreated controls).

stage are detected by Annexin $\mathrm{V}^{+} / \mathrm{PI}^{-}$staining, while cells in the late stages of apoptosis are marked by Annexin $\mathrm{V}^{+} / \mathrm{PI}^{+}$staining. In Fig. 3A and B, ganetespib significantly increased the early apoptotic cell population, as compared to the untreated control cells, in both AGS and N87 cell lines. Of note, the percentage of cells in late stage apoptosis was only significantly increased in AGS cells (Fig. 3B). Although ganetespib did not significantly modify the percentage of cells in late stage apoptosis in N87 cells, ganetespib-induced early stage apoptosis was more striking. Together, these results markedly demonstrate that ganetespib induces cell death via apoptosis in GC cell lines.

Caspases are the mediators of drug-induced cell death and ultimate dissociation. In order to identify the key mechanism of apoptosis-mediated cell death with ganetespib treatment, we next examined the activation of caspases and PARP in ganetespib-treated cells. As in Fig. 3C, ganetespib induced remarkable cleavage of PARP (in N87 cells only) and caspase-3, which are common proteolytic markers of apoptosis. Importantly, ganetespib treatment resulted in significant accumulation of cleaved caspase- 8 and caspase-9 (Fig. 3C), which are the apical caspases for death receptor and mitochondrial pathways, respectively. Moreover, ganetespib induced significant downregulation of survivin, an apoptotic antagonist, in both cell lines.

As we observed that ganetespib induced a significant increase in cleaved caspase- 8 and -9 without corresponding decrease in zymogen levels, we examined the mRNA levels of caspase-8, caspase- 9 and other apoptotic regulators, including Bcl-2, BIRC5 and MCL1. When the cells were treated with $50 \mathrm{nM}$ ganetespib for $16 \mathrm{~h}$, mRNA levels of caspase- 8 and -9 significantly increased and mRNA levels of Bcl-2 and BIRC5 significantly decreased in both cell lines (data not shown), suggesting that ganetespib regulates apoptosis at both transcriptional and translational levels. In all, these data indicate that ganetespib promotes the apoptotic cascade at multiple regulatory levels.

Ganetespib substantially suppresses RTK signaling. Deregulation of RTKs, such as ErbB2 and EGFR, plays a critical role in GC development, including cell proliferation, 
A

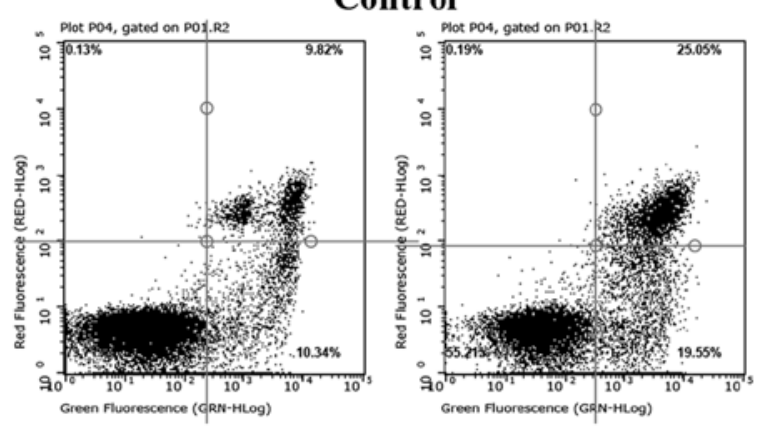

$25 \mathrm{nM}$

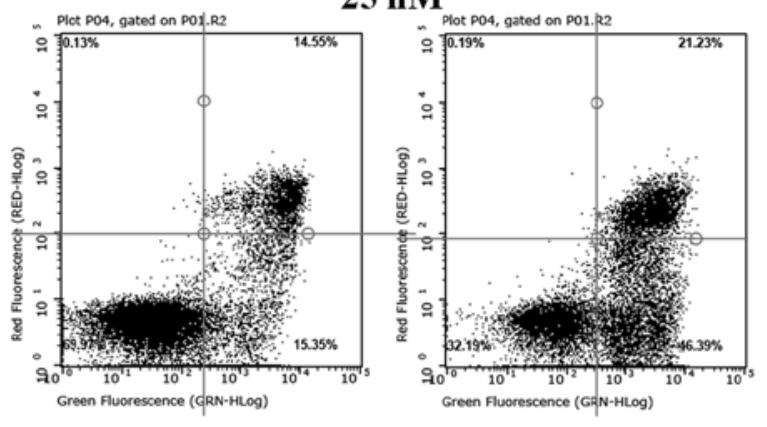

$50 \mathrm{nM}$
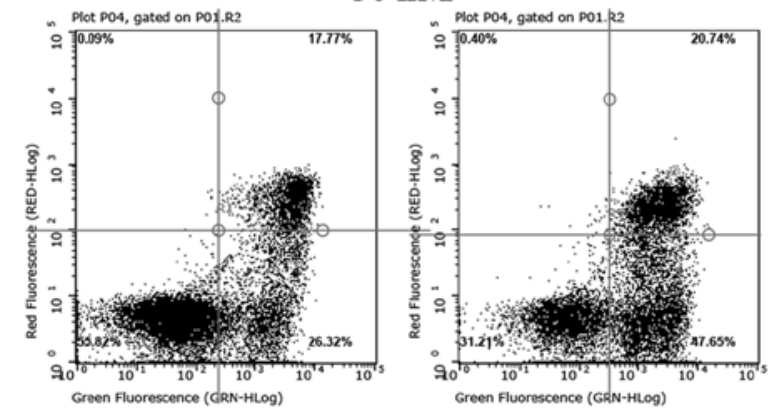

B

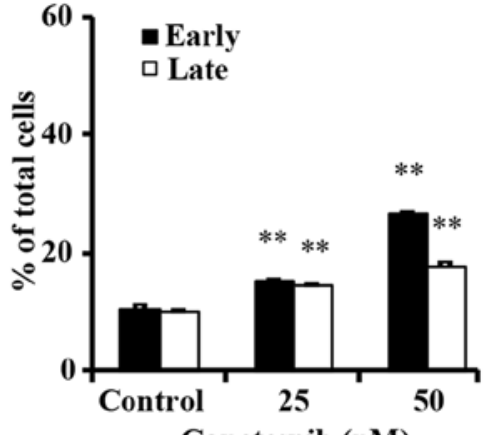

Ganetespib (nM)
N87

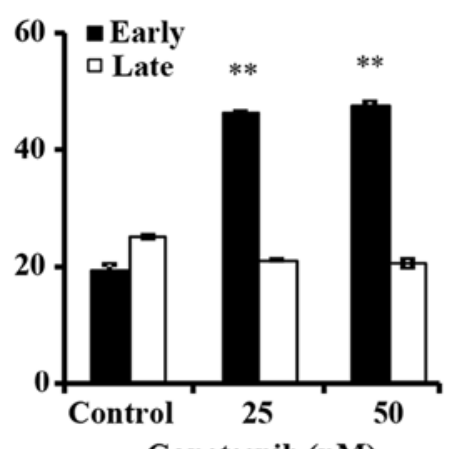

Ganetespib (nM)
C

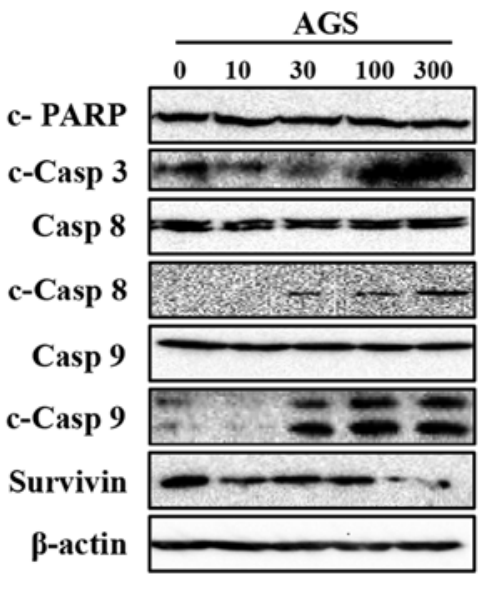

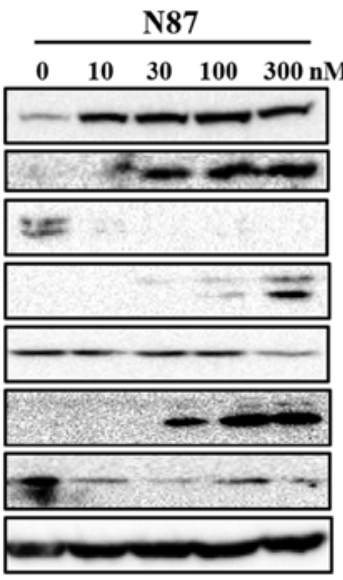

Figure 3. Ganetespib induces apoptosis in GC cells. (A and B) Apoptosis in AGS and N87 cells after treatment with ganetespib $(0,25$ or $50 \mathrm{nM})$ for $24 \mathrm{~h}$ was measured using Annexin V/PI staining. Representative flow cytometry plots are shown in (A). The percentage of cells in early (Annexin $\left.\mathrm{V}^{+} / \mathrm{PI}^{-}\right)$and late (Annexin $\left.\mathrm{V}^{+} / \mathrm{PI}^{+}\right)$apoptosis are graphed in $(\mathrm{B})$. Data are the means $\pm \mathrm{SEM}\left({ }^{* *} \mathrm{P} \leq 0.01\right.$ as compared to the corresponding untreated controls) for three replicates. (C) Ganetespib-treated $(0,10,30,100$ or $300 \mathrm{nM}$ for $24 \mathrm{~h})$ AGS and N87 cells were analyzed using western blotting of apoptotic markers.

angiogenesis and metastasis (8). The role of ganetespib in inhibiting EGFR-mediated GC was previously revealed by Liu et al (11). Therefore, we examined the effects of ganetespib (0-1000 nM) on the expression and activation of a series of markers representing different levels of the ErbB2 signaling cascade. We demonstrated that ganetespib remarkably downregulates the activation/phosphorylation of ErbB2 in N87 cells and its downstream effector molecules Erk, Akt, mTOR, GSK3 and Src, indicating the inhibitory effect of ganetespib on the kinase activities of the RTK pathway (Fig. 4A). Importantly, total protein levels of ErbB2, Akt, GSK3 and Src were also significantly downregulated in ganetespib-treated cells. Although AGS cells do not express ErbB2, ganetespib treatment significantly reduced the activation/phosphorylation of Erk, Akt, mTOR, GSK3 and Src also in this cell line. To confirm that RTK/ErbB2 signaling inhibition is a critical mechanism of ganetespib-induced cellular responses, we used lapatinib, an EGFR/ErbB2 dual inhibitor, to suppress EGFR and ErbB2 kinase activity. Lapatinib and ganetespib induced similar effects on Erk and Akt activation/phosphorylation (Fig. 4B), which indicates that the inhibition of RTK signaling is necessary for the actions of both drugs. Noteworthy, the combined treatment of ganetespib (30 or $100 \mathrm{nM})+$ lapatinib $(100 \mathrm{nM})$ synergistically enhanced the inhibition of Erk and Akt activation/phosphorylation. Thus, our data support that ganetespib effectively inhibits HSP90 client growth factors leading to RTK pathway inhibition and consequent cellular activities in GC cells.

\section{Discussion}

HSP90 inhibitors have gained much attention over the last few decades owing to their role in targeting HSP90 client proteins, including Akt, Raf, Erk, ErbB2 and EGFR, that are involved in various cancers (14). Due to solubility and toxicity issues, the first generation of geldanamycin-based HSP90 inhibitors were withdrawn from clinical trials, but they provided a proof of concept for targeting HSP90 in cancer. Promisingly, second generation HSP90 inhibitors exhibit more efficacy and less toxicity than the former inhibitors (15). In particular, 

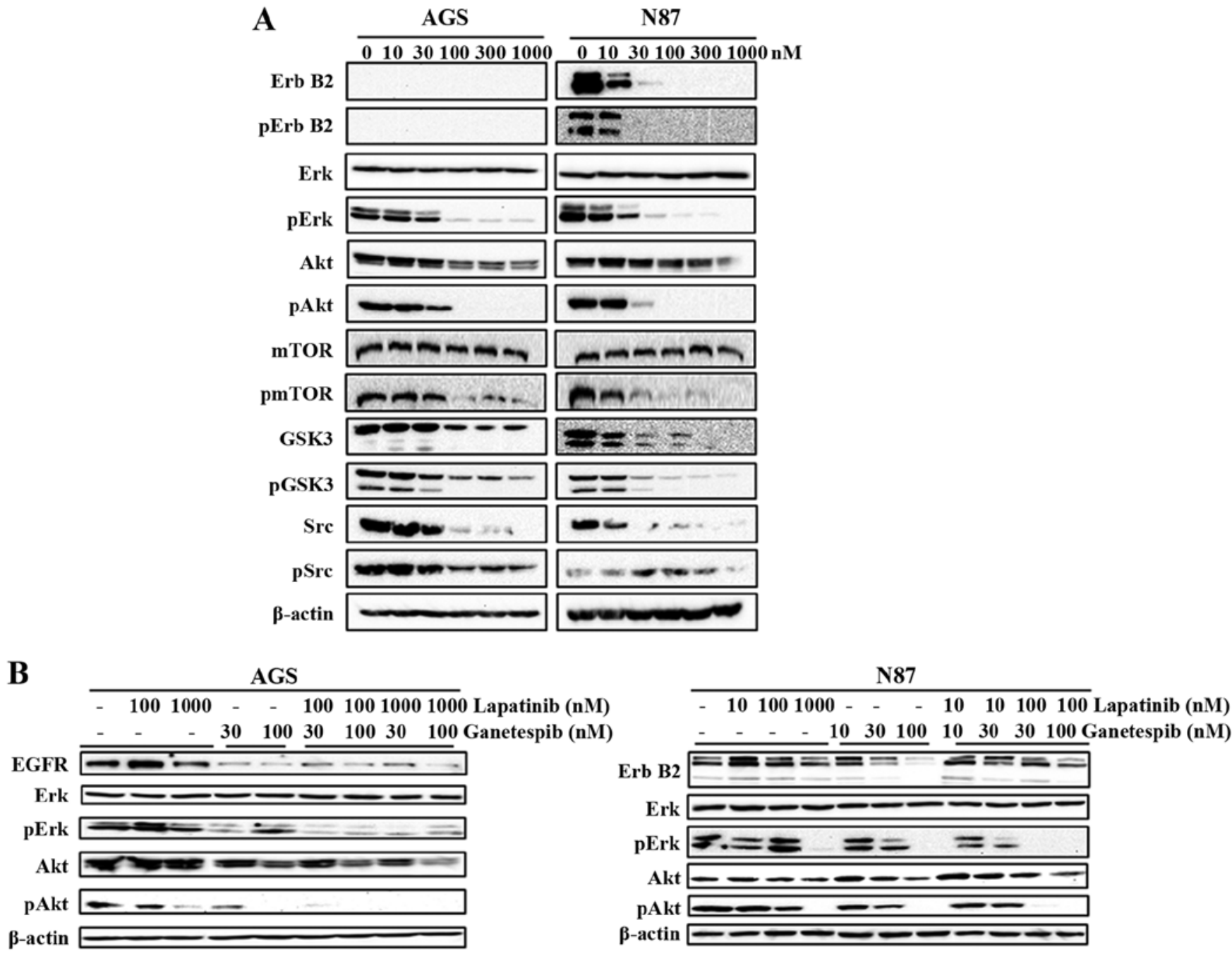

Figure 4. Ganetespib suppresses RTK signaling in GC cells. (A) AGS and N87 cells were treated with ganetespib ( $0,10,30,100,300 \mathrm{or} 1000 \mathrm{nM})$ for $16 \mathrm{~h}$ and then were analyzed for protein expression of key markers of the RTK pathways using western blotting. (B) AGS and N87 cells were also treated with lapatinib $(0,10,100$ or $1000 \mathrm{nM})+$ - ganetespib $(0,10,30$ or $100 \mathrm{nM})$ for $16 \mathrm{~h}$, followed by western blotting of the indicated RTK pathway markers.

ganetespib (STA-9090) is a potent resorcinol-based second generation HSP90 inhibitor with a unique triazolone moiety and improved efficacy and safety profile, without hepatotoxicity and ocular toxicity, than earlier HSP90 inhibitors. Preclinical studies involving ganetespib, alone or in combination with other drugs (16), reported improved efficacy and cytotoxicity in various hematological and solid tumors, including those with mutant kinases, such as B-RAF, EGFR and c-KIT $(9,17,18)$. The antitumor effects of ganetespib are attributed to its ability to inhibit cell growth via cell cycle arrest, apoptosis and PI3K/Akt, MAPK/Erk, mTOR and JAK2/STAT3 signaling inhibition (9).

In ErbB2-amplified breast cancer cell and xenograft models, ganetespib has demonstrated potential as a monotherapy or in combination with other drugs, such as trastuzumab, to prevent tumor resistance and regression (19). In addition, ganetespib has exhibited greater efficacy than first generation inhibitors in degrading HSP90 client proteins after short exposure (10). The ability of ganetespib to inhibit multiple targets of growth and survival pathways, especially the cell cycle and apoptosis, makes it an attractive strategy that can be used to prevent advanced GC, including ErbB2-amplified and mutant EGFR subtypes. Thus, in the present study, we investigated the effects of ganetespib treatment on AGS and N87 human GC cell lines. Our results determined that ganetespib inhibits cell proliferation (Fig. 1), induces G2/M cell cycle arrest (Fig. 2) and activates both cell death receptor- and mitochondria-mediated apoptotic pathways (Fig. 3), alongside inhibition of RTK and PI3K/Akt/MAPK signaling (Fig. 4) in AGS and N87 GC cells.

HSP90 client proteins include several proteins involved in cell cycle regulation and progression, such as Cdk1, Cdk4, cyclin D, cyclin B and survivin. These client proteins play important roles in driving mitogenic processes, as well as in G1/S, G2/M and spindle checkpoint controls (20). HSP90 inhibition can thus induce both G1/S and G2/M cell cycle arrest. The majority of the first generation HSP90 inhibitors have been reported to induce G1 cell cycle arrest in cancer cells. As such, geldanamycin and its derivative, 17-AAG, cause Rb-dependent G1 arrest in breast cancer cells, which is associated with downregulation of cyclin D (21). In our study, our data indicate that ganetespib inhibits cell proliferation by inducing cell cycle arrest in $\mathrm{G} 2 / \mathrm{M}$ phase with significant upregulation of cyclin B1 and p27 at both transcriptional and translational levels and concurrent downregulation of Cdk1, 
cyclin D1 and cyclin E proteins, CDK4 (mRNA) and transcription factor E2F1. Cyclin B1 and Cdk1 are major regulators of $\mathrm{G} 2 / \mathrm{M}$ cell cycle checkpoint and their interaction triggers the entry into the mitotic phase $(22,23)$. Although increased p27 levels are associated with G0/G1 arrest, we do not see an accumulation of cells in G0/G1 phase.

Earlier studies have shown that in mitogen-starved states, upregulated p27 can directly bind to cyclin B1 and block the progression through $\mathrm{G} 2 / \mathrm{M}$ phase $(22,24,25)$. Therefore, the overexpression of p27 that we observed in our study might be accompanied by the blocking of cyclin B1 to induce $\mathrm{G} 2 / \mathrm{M}$ arrest. Nevertheless, further studies are required to understand the role of elevated p27 and cyclin B1 levels in the current scenario. Liu et al recently reported that $\mathrm{G} 2 / \mathrm{M}$ cell cycle arrest in ganetespib-treated MGC-803 GC cells was associated with decreased cyclin B1, Cdk1 and Chk1 levels (11). Specifically, this ganetespib-induced reduction in cyclin $\mathrm{B} 1$ expression in MGC-803 cells is inconsistent with our findings in AGS and N87 GC cells (Fig. 2), suggesting cell line-specific effects of ganetespib on cell cycle regulation. Thus, we show that ganetespib induces G2/M cell cycle arrest in AGS and N87 cell lines with concomitant increases in cyclin B1 and p27 levels. Additional studies are required to fully understand the potential cell-specific mechanisms of ganetespib.

The cell cycle arrest induced by most HSP90 inhibitors advances the cells towards programmed cell death (20). In this study, we found that ganetespib-induced G2/M cell cycle arrest initiated a signaling cascade leading to apoptosis in both AGS and N87 cell lines. Our results demonstrated a dose-dependent increase in early phase apoptosis in AGS and N87 cell lines and an increasing trend for late phase apoptosis in AGS cells, but not in N87 cells (Fig. 3). Since $\mathrm{PI}^{+}$cells can also be undergoing necrosis (26), late phase apoptosis may not be the most accurate indicator of continued apoptosis in cells. To further investigate the mechanism of ganetespibinduced apoptosis, we evaluated different proteins involved in caspase-mediated apoptotic pathways. Our results showed an increase in cleaved caspase- $-3,-8$ and -9 along with cleaved PARP (only in N87 cells), which are indicative of the activation of both cell death receptor- and mitochondria-mediated apoptotic pathways. Also, survivin, an anti-apoptotic marker, is decreased in both cell lines, which provides additional evidence of apoptotic induction. The variation in the c-PARP levels between the AGS and N87 cell lines tested in our study could be due to the short treatment time of ganetespib (24 h) as compared to earlier reports that showed increased c-PARP levels after 48 or $72 \mathrm{~h}$ of treatment (11). Thus, our data also suggest the increased sensitivity of N87 cells, as compared to AGS cells, to ganetespib after a short exposure time. Taken together, our results support the activation of both cell death receptor- and mitochondria-mediated apoptotic pathways in ganetespib-treated GC cells.

RTK-mediated signaling pathways are activated downstream of various HSP90 client proteins, including ErbB2, EGFR and VEGF, and are thus sensitive to HSP90 inhibition. These pathways, including PI3K/Akt and MAPK/Erk, are effectively suppressed by various HSP90 inhibitors [such as 17-DMAG (27), NVP-AUY922 (19) and LD053 (28)] thereby inhibiting targets associated with cell proliferation, survival and invasion (8). Our study revealed that ganetespib treatment in GC cells (ErbB2 expression was only detected in N87 cells and not detected in AGS cells) downregulates both ErbB2 activation/phosphorylation and its downstream signaling effector molecules, including Erk, Akt, mTOR, GSK3 and Src (Fig. 4). ErbB2 is one of the most sensitive client proteins of HSP90, making various HSP90 inhibitors remarkably effective in downregulating ErbB2-mediated signaling.

NVP-AUY922 is a potent HSP90 inhibitor that has shown efficacy in preclinical models of ErbB2-amplified, trastuzumab-resistant GC by degrading ErbB2, Akt and Erk and subsequently suppressing downstream Akt-mediated signaling $(19,29)$. Similarly, another HSP90 inhibitor, LD053, exerts its anti-proliferative effects by promoting the dissociation of the HSP90-Cdc37 complex, degrading c-Raf and inhibiting Akt-mediated c-Raf/Mek/Erk and PI3K/Akt signaling (28). Ganetespib is also shown to inhibit HSP90-p23 complex formation, thereby inhibiting HSP90-driven client proteins, including mutant EGFR, mutant ErbB2, KRAS and associated downstream signaling pathways in mutant ErbB2-driven non-small cell lung cancers (30). EGFR-mediated inhibition of PI3K/Akt, Ras/Raf/Erk and JAK/STAT pathways was also reported in GC cell and xenograft models (11). Our results further support these data and demonstrate inhibition of PI3K/ Akt and MAPK/Erk signaling downstream of EGFR and ErbB2 in GC cells, suggesting that the signaling regulation by ganetespib may target both EGFR- and ErbB2-mediated pathways and subsequently modulate the associated downstream oncogenic signaling.

Despite multiple clinical trials that have reported a promising safety profile for ganetespib in the treatment of non-small cell lung cancer and metastatic breast cancer, the clinical application of ganetespib remains uncertain due to marginal changes in patient outcomes (31-33). Nevertheless, in support of our current study and previous reports indicating that EGFR and/or ErbB2-overexpressing cancer subtypes may be more responsive to ganetespib, an ongoing phase I clinical trial is designed to test ganetespib in combination with other therapeutic agents in ErbB2 ${ }^{+}$metastatic breast cancer patients (ClinicalTrials.gov Identifier: NCT02060253). To further extend the clinical potential of ganetespib, our study helps to form the foundation for future preclinical studies and ultimately clinical trials exploring the application of ganetespib as a monotherapy or combinational therapy for $\mathrm{EGFR}^{+} / \mathrm{ErbB}^{+}$ gastric cancer patients.

Overall, our studies in AGS and N87 human cell line models of GC indicate the effectiveness of ganetespib in inhibiting proliferation and colony-forming ability of cells by inducing G2/M cell cycle arrest and apoptosis. The anticancer capacity of ganetespib was further corroborated by the inhibition of ErbB2/RTK and downstream PI3K/Akt/ MAPK signaling pathways. Thus, our data provide additional mechanistic insights into the activity of ganetespib and support its clinical development for effective treatment of $\mathrm{EGFR}^{+} / \mathrm{ErbB}^{+} \mathrm{GC}$.

\section{Acknowledgements}

We thank Dr Erin Howard for critical reading and editing of the manuscript. This work was supported in part by the American Cancer Society (grant no. RSG-08-138-01-CNE), 
the National Institute of Environmental Health Sciences (grant no. R21ES025337), the National Cancer Institute (grant no. 5U54CA156735), the National Institute on Alcohol Abuse and Alcoholism (grant no. U54 AA019765), and a UNC GA Research Opportunities Initiative (ROI) Grant to X.Y.

\section{References}

1. Okamoto W, Okamoto I, Yoshida T, Okamoto K, Takezawa K, Hatashita E, Yamada Y, Kuwata K, Arao T, Yanagihara K, et al: Identification of c-Src as a potential therapeutic target for gastric cancer and of MET activation as a cause of resistance to c-Src inhibition. Mol Cancer Ther 9: 1188-1197, 2010.

2. American Cancer Society: Cancer Facts and Figures 2016. ACS Atlanta, GA, 2016. http://www.cancer.org/research/cancer-factsstatistics/all-cancer-facts-figures/cancer-facts-figures-2016.html. Accessed date January 24, 2017.

3. Hartgrink HH, Jansen EP, van Grieken NC and van de Velde CJ: Gastric cancer. Lancet 374: 477-490, 2009.

4. Yasui W, Hata J, Yokozaki H, Nakatani H, Ochiai A, Ito H and Tahara E: Interaction between epidermal growth factor and its receptor in progression of human gastric carcinoma. Int $\mathbf{J}$ Cancer 41: 211-217, 1988.

5. Mi L, Ji X and Ji J: Prognostic biomarker in advanced gastric cancer. Transl Gastrointest Cancer 5: 16-29, 2016.

6. Aydin K, Okutur SK, Bozkurt M, Turkmen I, Namal E, Pilanci K, Ozturk A, Akcali Z, Dogusoy G and Demir OG: Effect of epidermal growth factor receptor status on the outcomes of patients with metastatic gastric cancer: A pilot study. Oncol Lett 7: 255-259, 2014.

7. Wadhwa R, Song S, Lee JS, Yao Y, Wei Q and Ajani JA: Gastric cancer-molecular and clinical dimensions. Nat Rev Clin Oncol 10: 643-655, 2013.

8. Moser C, Lang SA and Stoeltzing O: Heat-shock protein 90 (Hsp90) as a molecular target for therapy of gastrointestinal cancer. Anticancer Res 29: 2031-2042, 2009.

9. Wang Y, Trepel JB, Neckers LM and Giaccone G: STA-9090, a small-molecule Hsp90 inhibitor for the potential treatment of cancer. Curr Opin Investig Drugs 11: 1466-1476, 2010.

10. Jhaveri K and Modi S: Ganetespib: Research and clinical development. Onco Targets Ther 8: 1849-1858, 2015.

11. Liu H, Lu J, Hua Y, Zhang P, Liang Z, Ruan L, Lian C, Shi H, Chen K and Tu Z: Targeting heat-shock protein 90 with ganetespib for molecularly targeted therapy of gastric cancer. Cell Death Dis 6: e1595, 2015.

12. Mahata S, Maru S, Shukla S, Pandey A, Mugesh G, Das BC and Bharti AC: Anticancer property of Bryophyllum pinnata (Lam.) Oken. leaf on human cervical cancer cells. BMC Complement Altern Med 12: 15, 2012.

13. Takayama S, Reed JC and Homma S: Heat-shock proteins as regulators of apoptosis. Oncogene 22: 9041-9047, 2003.

14. Jhaveri K, Ochiana SO, Dunphy MP, Gerecitano JF, Corben AD, Peter RI, Janjigian YY, Gomes-DaGama EM, Koren J III, Modi S, et al: Heat shock protein 90 inhibitors in the treatment of cancer: Current status and future directions. Expert Opin Investig Drugs 23: 611-628, 2014.

15. Den RB and Lu B: Heat shock protein 90 inhibition: Rationale and clinical potential. Ther Adv Med Oncol 4: 211-218, 2012.

16. Lai CH, Park KS, Lee DH, Alberobello AT, Raffeld M, Pierobon M, Pin E, Petricoin Iii EF, Wang Y and Giaccone G: HSP-90 inhibitor ganetespib is synergistic with doxorubicin in small cell lung cancer. Oncogene 33: 4867-4876, 2014.

17. Smith DL, Acquaviva J, Sequeira M, Jimenez JP, Zhang C, Sang J, Bates RC and Proia DA: The HSP90 inhibitor ganetespib potentiates the antitumor activity of EGFR tyrosine kinase inhibition in mutant and wild-type non-small cell lung cancer. Target Oncol 10: 235-245, 2015.
18. Acquaviva J, Smith DL, Jimenez JP, Zhang C, Sequeira M, He S Sang J, Bates RC and Proia DA: Overcoming acquired BRAF inhibitor resistance in melanoma via targeted inhibition of $\mathrm{Hsp} 90$ with ganetespib. Mol Cancer Ther 13: 353-363, 2014.

19. Wainberg ZA, Anghel A, Rogers AM, Desai AJ, Kalous O, Conklin D, Ayala R, O'Brien NA, Quadt C, Akimov M, et al: Inhibition of HSP90 with AUY922 induces synergy in HER2amplified trastuzumab-resistant breast and gastric cancer. Mol Cancer Ther 12: 509-519, 2013.

20. Burrows F, Zhang H and Kamal A: Hsp90 activation and cell cycle regulation. Cell Cycle 3: 1530-1536, 2004.

21. Srethapakdi M, Liu F, Tavorath R and Rosen N: Inhibition of Hsp90 function by ansamycins causes retinoblastoma gene product-dependent G1 arrest. Cancer Res 60: 3940-3946, 2000.

22. Foijer F and te Riele H: Check, double check: The G2 barrier to cancer. Cell Cycle 5: 831-836, 2006.

23. DiPaola RS: To arrest or not to G(2)-M Cell-cycle arrest: commentary re: A.K. Tyagi et al., Silibinin strongly synergizes human prostate carcinoma DU145 cells to doxorubicin-induced growth inhibition, $\mathrm{G}(2)-\mathrm{M}$ arrest, and apoptosis. Clin. Cancer Res. 8: 3512-3519, 2002. Clin Cancer Res 8: 3311-3314, 2002.

24. Toyoshima $\mathrm{H}$ and Hunter T: p27, a novel inhibitor of G1 cyclinCdk protein kinase activity, is related to p21. Cell 78: 67-74, 1994.

25. Foijer F, Delzenne-Goette E, Dekker M and Te Riele H: In vivo significance of the $\mathrm{G} 2$ restriction point. Cancer Res 67: 9244-9247, 2007.

26. Sawai H and Domae N: Discrimination between primary necrosis and apoptosis by necrostatin-1 in Annexin V-positive/propidium iodide-negative cells. Biochem Biophys Res Commun 411: 569-573, 2011.

27. Lang SA, Klein D, Moser C, Gaumann A, Glockzin G, Dahlke MH, Dietmaier W, Bolder U, Schlitt HJ, Geissler EK, et al: Inhibition of heat shock protein 90 impairs epidermal growth factor-mediated signaling in gastric cancer cells and reduces tumor growth and vascularization in vivo. Mol Cancer Ther 6: 1123-1132, 2007.

28. Lu C, Liu D, Jin J, Deokar H, Zhang Y, Buolamwini JK, Yu X, Yan $C$ and Chen $X$ : Inhibition of gastric tumor growth by a novel Hsp90 inhibitor. Biochem Pharmacol 85: 1246-1256, 2013.

29. Lee KH, Lee JH, Han SW, Im SA, Kim TY, Oh DY and Bang YJ: Antitumor activity of NVP-AUY922, a novel heat shock protein 90 inhibitor, in human gastric cancer cells is mediated through proteasomal degradation of client proteins. Cancer Sci 102: 1388-1395, 2011.

30. Shimamura T, Perera SA, Foley KP, Sang J, Rodig SJ, Inoue T, Chen L, Li D, Carretero J, Li YC, et al: Ganetespib (STA-9090), a nongeldanamycin HSP90 inhibitor, has potent antitumor activity in in vitro and in vivo models of non-small cell lung cancer. Clin Cancer Res 18: 4973-4985, 2012.

31. Socinski MA, Goldman J, El-Hariry I, Koczywas M, Vukovic V, Horn L, Paschold E, Salgia R, West H, Sequist LV, et al: A multicenter phase II study of ganetespib monotherapy in patients with genotypically defined advanced non-small cell lung cancer. Clin Cancer Res 19: 3068-3077, 2013.

32. Ramalingam S, Goss G, Rosell R, Schmid-Bindert G, Zaric B, Andric Z, Bondarenko I, Komov D, Ceric T, Khuri F, et al: A randomized phase II study of ganetespib, a heat shock protein 90 inhibitor, in combination with docetaxel in second-line therapy of advanced non-small cell lung cancer (GALAXY-1). Ann Oncol 26: 1741-1748, 2015 .

33. Jhaveri K, Chandarlapaty S, Lake D, Gilewski T, Robson M, Goldfarb S, Drullinsky P, Sugarman S, Wasserheit-Leiblich C, Fasano J, et al: A phase II open-label study of ganetespib, a novel heat shock protein 90 inhibitor for patients with metastatic breast cancer. Clin Breast Cancer 14: 154-160, 2014. 